\title{
An orthogonal spectrum sharing scheme for wireless sensor networks
}

\author{
Vivek A Bohara ${ }^{1 *}$, See Ho Ting ${ }^{1}$, Yang Han ${ }^{1}$ and Ashish Pandharipande ${ }^{2}$
}

\begin{abstract}
It is not economically viable to allocate a dedicated spectrum band to wireless sensor networks (WSNs). Moreover, sharing a spectrum band with incumbent (primary) system compromises the reliability and performance of both the systems due to interference from one system to another. In this article, we address this limitation by proposing a two-phase orthogonal spectrum sharing protocol for a WSN which exploits multiple sensor nodes to effectively cancel out the interference from a WSN to the primary system, and vice versa. As a consequence, it is possible to achieve spectrum access for the WSN without compromising on the performance of either systems. Performance of WSN as well as the primary system is quantified in terms of average received signal to noise ratio. We then validate the efficiency of the proposed scheme through analytical and simulation results.
\end{abstract}

\section{Introduction}

Recently, wireless sensor networks (WSNs) [1-5] are being increasingly deployed all over the world at an accelerated pace. This has been made practically feasible by significant advances in microelectro-mechanical systems (MEMS) technology, radio communications and digital electronics [2]. A typical WSN consists of spatially distributed sensor nodes deployed in an ad hoc manner which collects data and pass on to a central base station (CBS) via a radio link. The CBS can be a $\mathrm{PC}$, data server, dedicated monitoring device, or any other gateway to a higher data rate device. WSNs are used for various applications including military surveillance, habitat monitoring, object tracking, traffic monitoring, etc.

Most of the sensor nodes are autonomous and send data over the radio link only when required. Furthermore, there is an increasing trend of deploying WSN in urban areas as part of the infrastructure to support smart building initiatives and power meter readings for smart grids, to name a few. However, radio spectrum in urban areas are generally extremely crowded as evident from the National Telecommunications and Information Administrations (NTIA) frequency allocation chart ${ }^{1}$ and

\footnotetext{
* Correspondence: vive0006@e.ntu.edu.sg

'School of Electrical and Electronic Engineering, Nanyang Technological

University, Singapore, 639801 Singapore

Full list of author information is available at the end of the article
}

thus it is not possible nor economically viable to allocate a dedicated radio spectrum band to a WSN.

Factors such as the above have spurred the demand for alternative spectrum access techniques for WSNs $[6,7]$. This demand has been further compounded by the inefficient usage of the licensed bands by the incumbent (primary) systems [8]. Researchers over the years have proposed dynamic spectrum access (DSA) techniques to utilize the spectrum more efficiently by allowing a secondary system (for example a WSN) to co-exist in the same frequency band as a primary system and opportunistically access the licensed bands [9-11]. But most of this techniques are interference limited, and the performance of the systems are limited by the amount of interference acceptable from one system to another [12-16].

In this article, by taking the above factors into consideration, we propose an orthogonal spectrum sharing scheme (OSSS) which allows a WSN to gain spectrum access along with a primary system without causing any interference to one another. As a result, the performance of primary system is not limited by the interference from WSN and vice versa. In the proposed scheme, a WSN, henceforth known as secondary system, is assumed to be a single-hop network with every sensor node being able to directly communicate with every other node. Secondary transmitters (STs) are spatially distributed sensor nodes that cooperatively monitor their physical environmental conditions and send an

\section{SpringerOpen ${ }^{\circ}$}

(c) 2011 Bohara et al; licensee Springer. This is an Open Access article distributed under the terms of the Creative Commons Attribution License (http://creativecommons.org/licenses/by/2.0), which permits unrestricted use, distribution, and reproduction in any medium, provided the original work is properly cited. 
update to their CBS, which for simplicity will be denoted as secondary receiver (SR). STs can communicate with each other in real time and the communication link between them can be formed by using a radio, infrared or an optical media depending upon the availability [2]. This inter-node communication helps in status monitoring of the STs and also avoids duplication of data at SR. Moreover, it also keeps all STs well informed of the latest information being sent to SR.

Under the proposed framework, the secondary system operates in the same frequency band as an incumbent primary system, which comprises of primary transmitter (PT) and primary receiver (PR). A higher priority is given to the primary system and the secondary system operates on a lower priority with a constraint that its operation does not affect the performance of primary system. For ease of analysis, we limit ourselves to two ST nodes, ST (1) and ST(2) and denote them as a ST cluster or simply ST wherever necessary. Do note that due to inter-node communication, ST(1) and ST(2) has access to the same sensor information that is to be sent to SR.

Cooperation techniques to enhance the performance of a communication system in terms of diversity, coverage extension, etc, have been studied extensively in literature [17-21]. Control signalling for practical cooperation schemes have also been proposed in [22-28]. In our proposed scheme, we presume that the primary system is an advanced system with a relaying functionality, like IEEE 802.16j [29], and it employs a practical handshake mechanism for cooperative relaying [27].

Consider a scenario in which the average signal to noise ratio (SNR) between PT and PR drops below a particular threshold. PT will seek cooperation from neighboring terminals to enhance its transmission performance by broadcasting a cooperative right-to-send (CRTS) message which also indicates the target average $\mathrm{SNR}, \overline{\mathrm{SNR}}_{\mathrm{T}}$, for the primary system. PR responds to CRTS by transmitting a cooperative clear-to-send (CCTS) message. Upon overhearing CRTS and CCTS, ST decides ${ }^{2}$ whether $\overline{\mathrm{SNR}}_{\mathrm{T}}$ can be met if it serves as an amplify-and-forward (AF) relay for the primary system. If yes, $\mathrm{ST}(2)$ responds by sending a cooperative clear-tohelp $(\mathrm{CCTH})$ message to $\mathrm{PT}$ and $\mathrm{PR}$, and the primary system correspondingly switches to a two-phase AF relaying transmission mode, with $\mathrm{ST}(1)$ acting as the primary relay. However, if $\mathbf{S T}$ is not able to assist the primary system to achieve $\overline{\mathrm{SNR}}_{\mathrm{T}}$, it will simply remain silent.

Once $\mathbf{S T}$ is confirmed as a relay, secondary spectrum access is achieved by adopting the following two-phase transmission protocol. The system models for the 1st and 2nd phase are shown in Figures 1 and 2, respectively. In the 1st phase, the primary signal transmitted by PT to PR is overheard by ST(1) and SR. Simultaneously in the

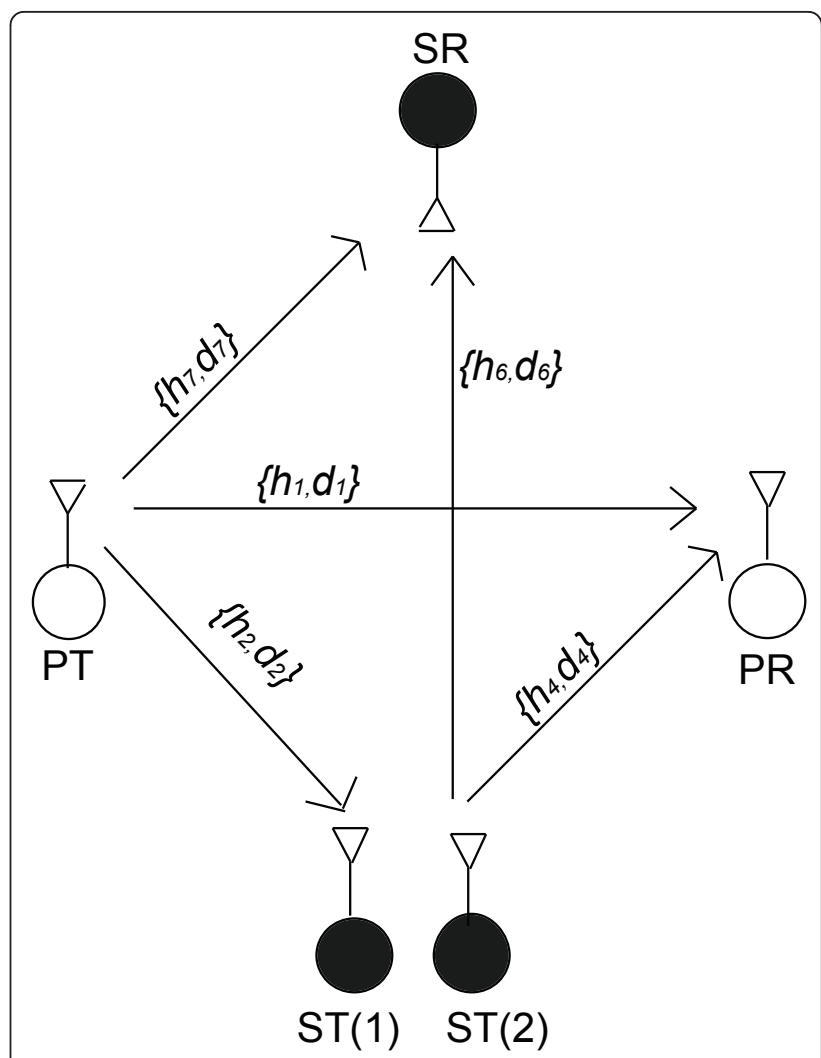

Figure 1 OSSS: 1st transmission phase

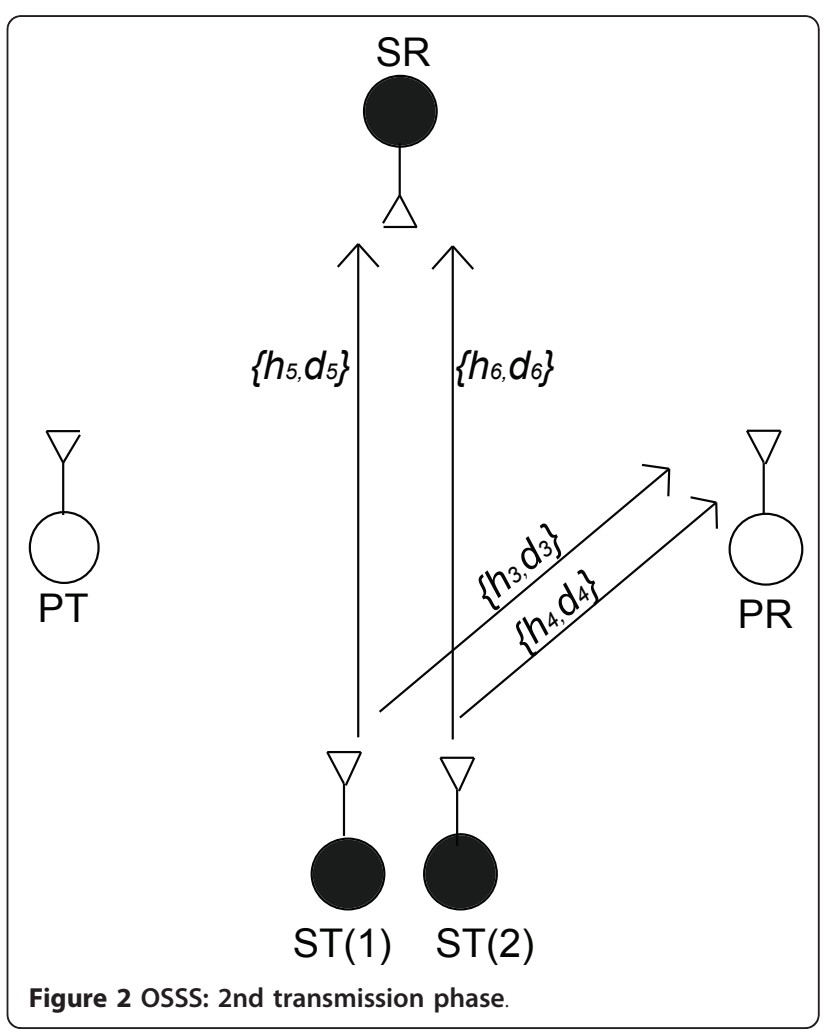


same phase, ST(2) transmits the secondary signal which is received by SR as well as PR. At ST(1), the primary signal received in the 1st phase is amplified according to its power constraint.

The 2nd phase of the proposed scheme is similar to a space time block code (STBC) design [30]. ST(1) and ST(2) transmit the negative complex conjugate of the amplified primary signal and complex conjugate of the secondary signal, respectively. At PR, the received signals after the twophase transmission are multiplied by an orthogonalization vector to cancel out the interference due to secondary signal and retrieve the primary signal. The secondary signal is retrieved at SR in the same way.

The most important attribute of the proposed scheme is that it is not interference-limited because of the orthogonality between the received primary and secondary signals. As a result, the performance of primary (secondary) system is not limited by the interference from secondary (primary) system. As shown later in this article, the secondary user is able to achieve spectrum access as long as it is willing to increase its transmit power such that $\overline{\mathrm{SNR}}_{\mathrm{T}}$ is met. This ability to trade-off transmit power with spectrum access opportunity is an attractive feature for WSNs as it allows the sensor nodes to maintain its Quality of Service (QoS), such as delay constraints. Another point to note is that although the proposed scheme has been illustrated by using WSN as a secondary system, the obtained analytical and performance results are also applicable to any radio (secondary user) that is interested in accessing the licensed spectrum as long as it does not compromise the performance of licensee ${ }^{3}$.

As a basic requirement for the proposed scheme, we assume that the primary system supports STBC [31] and the necessary channel state information (CSI) needed at the receiving terminals can be obtained through standard pilot symbol-aided channel estimation methods [32-34]. We analyze the above proposed scheme, henceforth called as orthogonal spectrum sharing scheme (OSSS), by deriving the closed-form expressions for average SNR of the primary system. For comparison, we also consider an interference limited scheme where ST uses AF with superposition coding (AF-SC) [35]. We show that for the same $\overline{\mathrm{SNR}}_{\mathrm{T}}$ requested by the primary system, OSSS can achieve a much higher performance for the secondary system than AF-SC.

The remainder of this article is organized as follows. Section 2 discusses the system model for OSSS and gives the general protocol description. Sections 3 and 4 present the analysis for OSSS and AF-SC schemes, respectively. Section 5 provides the simulation results. Finally, Section 6 concludes the article. The following notations are used in this article. $\mathrm{E}[\cdot]$ denotes the statistical expectation operator and a complex Gaussian random variable $z$ with mean $\mu$ and variance $\sigma^{2}$ is denoted as $z \sim C \mathcal{N}\left(\mu, \sigma^{2}\right)$. An exponential distributed random variable $x$ with mean $\frac{1}{\lambda}$ is denoted as $x \sim \varepsilon(\lambda)$ We denote the transpose and conjugate transpose of matrix $A$ as $A^{\mathrm{T}}$ and $A^{\mathrm{H}}$, respectively.

\section{System model and protocol description}

\section{System model}

The system model under consideration for the 1st and 2nd transmission phase is shown in Figures 1 and 2, respectively. The channel between all the links, i.e., PTPR, PT-ST(1), ST(1)-PR, ST(2)-PR, ST(1)-SR, ST(2)-SR, and PT-SR are modeled as Rayleigh flat fading with channel coefficients $h_{1}, h_{2}, h_{3}, h_{4}, h_{5}, h_{6}$, and $h_{7}$, respectively, thus $h_{i} \sim \mathcal{C N}\left(0, d_{i}^{-v}\right), i=1,2,3,4,5,6,7$ where $v$ is the path loss component and $d_{i}$ is the distance between the respective transmitters and receivers. Thus, all the links between the terminals can be characterized by the set of parameters $\left\{h_{i}, d_{i}\right\}$ as shown in Figures 1 and 2 . The instantaneous channel gain of each link is denoted by $\gamma_{i}=\left|h_{i}\right|^{2}$. The primary and secondary signals are denoted by $x_{\mathrm{p}}$ and $x_{\mathrm{s}}$, respectively, have zero mean and $\mathrm{E}\left[x_{\mathrm{p}}^{*} x_{\mathrm{p}}\right]=1, \mathrm{E}\left[x_{\mathrm{s}}^{*} x_{\mathrm{s}}\right]=1$. We denote the transmit power at PT and $\mathbf{S T}$ as $P_{\mathrm{p}}$ and $P_{\mathrm{s}}$, respectively.

\section{Protocol description}

In the situation where only the primary system is operating, i.e., there is no spectrum sharing, the average received SNR between $\mathrm{PT}$ and $\mathrm{PR}$ is given by

$$
\overline{\mathrm{SNR}}_{\mathrm{d}}=\mathrm{E}\left[\frac{P_{\mathrm{p}} \gamma_{1}}{\sigma^{2}}\right]=\frac{P_{\mathrm{p}}}{d_{1}^{v} \sigma^{2}}
$$

where $\sigma^{2}$ is the variance of additive white Gaussian noise (AWGN) at PR. The following steps illustrate the control signalling involved.

(1) PT obtains $\overline{\mathrm{SNR}}_{\mathrm{d}}$ from PR through conventional channel quality feedback mechanism [36] and checks whether $\overline{\mathrm{SNR}}_{\mathrm{d}}<\overline{\mathrm{SNR}}_{\mathrm{T}}$. If yes, go to step 2. Otherwise continue with the ongoing transmission.

(2) PT checks whether a retransmission of the same signal as part of an ARQ protocol will assist in achieving $\overline{\mathrm{SNR}}_{\mathrm{T}}$, i.e.,

$\overline{\mathrm{SNR}}_{\mathrm{MRC}} \geq \overline{\mathrm{SNR}}_{\mathrm{T}}$

where $\overline{\mathrm{SNR}}_{\mathrm{MRC}}=\frac{2 P_{\mathrm{p}}}{d_{1}^{v} \sigma^{2}}$ is the average received SNR for the primary system after the retransmission with maximum ratio combining (MRC) at PR. If yes, PT proceeds with ARQ protocol. Otherwise, go to step 3. 
(3) PT transmits CRTS which indicates $\overline{\mathrm{SNR}}_{\mathrm{T}}$ required by the primary system and PR responds by sending CCTS.

(4) Upon overhearing CRTS and CCTS from PT and $\mathrm{PR}$, respectively, $\mathbf{S T}$ will decide whether it is able to assist the primary system in achieving $\overline{\mathrm{SNR}}_{\mathrm{T}}$ by calculating $\overline{\mathrm{SNR}}_{\mathrm{p}}$, which is the achievable average received SNR of the primary system with OSSS. If $\overline{\mathrm{SNR}}_{\mathrm{p}} \geq \overline{\mathrm{SNR}}_{\mathrm{T}}$, then ST(2) will broadcast CCTH, and the primary system correspondingly switches to the two-phase OSSS protocol. Otherwise, ST will simply remain silent.

\section{Average received SNR for OSSS}

\section{Average received SNR of primary system with OSSS}

1) Phase 1: In the 1st transmission phase, as shown in Figure 1 , the primary signal $x_{\mathrm{p}}$ is transmitted by PT and secondary signal $x_{\mathrm{S}}$ is transmitted by ST(2) simultaneously. Denoting the signals received by PR, SR and $\mathrm{ST}(1)$ as $\gamma_{\mathrm{pr}}^{(1)}, \gamma_{\mathrm{sr}}^{(1)}$ and $y_{\mathrm{s} \text {, }}$ respectively, we have, ${ }^{4}$

$$
\begin{aligned}
& y_{\mathrm{pr}}^{(1)}=\sqrt{P_{\mathrm{p}}} h_{1} x_{\mathrm{p}}+\sqrt{P_{\mathrm{s}}} h_{4} x_{\mathrm{s}}+n_{11}, \\
& y_{\mathrm{sr}}^{(1)}=\sqrt{P_{\mathrm{p}}} h_{7} x_{\mathrm{p}}+\sqrt{P_{\mathrm{s}}} h_{6} x_{\mathrm{s}}+n_{12}, \\
& y_{\mathrm{st}}=\sqrt{P_{\mathrm{p}}} h_{2} x_{\mathrm{p}}+n_{13} .
\end{aligned}
$$

Here $n_{1 j} \sim \mathcal{C N}\left(0, \sigma^{2}\right), j=1,2,3$ is the AWGN at the respective receivers in the 1 st transmission phase.

2) Phase 2: Let $z_{\mathrm{s}}^{(1)}$ and $z_{\mathrm{s}}^{(2)}$ be the transmitted signals from ST(1) and ST(2) during the $2^{\text {nd }}$ phase, respectively. The transmitted signal vector in the 2nd phase from ST can then be written as

$$
z_{\mathrm{s}}=\left[\begin{array}{cc}
g & 0 \\
0 & \sqrt{\frac{P_{\mathrm{s}}}{2}}
\end{array}\right] x_{\mathrm{st}}
$$

where $\quad z_{\mathrm{s}}=\left[\begin{array}{ll}z_{\mathrm{s}}^{(1)} & z_{\mathrm{s}}^{(2)}\end{array}\right]^{\mathrm{T}}, \quad x_{\mathrm{st}}=\left[-\gamma_{\mathrm{st}}^{*} x_{\mathrm{s}}^{*}\right]^{\mathrm{T}} \quad$ and $g=\sqrt{\frac{P s}{2\left(P_{\mathrm{p}} \gamma_{2}+\sigma^{2}\right)}}$. The signal received at PR in the 2nd phase is thus,

$$
y_{\mathrm{pr}}^{(2)}=h_{\mathrm{p}} z_{\mathrm{s}}+n_{21}
$$

where $\boldsymbol{h}_{\mathrm{p}}=\left[\begin{array}{ll}h_{3} & h_{4}\end{array}\right]$ and $n_{21} \sim \mathcal{C N}\left(0, \sigma^{2}\right)$ is the AWGN. Taking the complex conjugate of (7) at PR we obtain,

$$
\begin{aligned}
y_{\mathrm{pr}}^{(2) *} & =\left(\boldsymbol{h}_{\mathrm{p}} z_{\mathrm{s}}\right)^{*}+n_{21}^{*} \\
& =\sqrt{\frac{P_{\mathrm{s}}}{2}} h_{4}^{*} x_{\mathrm{s}}-g h_{3}^{*} y_{\mathrm{st}}+n_{21}^{*} \\
& =\sqrt{\frac{P_{\mathrm{s}}}{2}} h_{4}^{*} x_{\mathrm{s}}-g h_{3}^{*} \sqrt{P_{\mathrm{p}}} h_{2} x_{\mathrm{p}}-n_{3} .
\end{aligned}
$$

where $n_{3}=g h_{3}^{*} n_{13}-n_{21}^{*}$. Thus, the signal at PR after the two-phase transmission can be written as

$$
y_{\mathrm{p}}=H_{\mathrm{p}} \boldsymbol{x}+\boldsymbol{n}_{\mathrm{p}}
$$

where $\boldsymbol{y}_{\mathrm{p}}=\left[\begin{array}{ll}y_{\mathrm{pr}}^{(1)} & y_{\mathrm{pr}}^{(2) *}\end{array}\right]^{\mathrm{T}}, \boldsymbol{x}=\left[\begin{array}{ll}x_{\mathrm{p}} & x_{\mathrm{s}}\end{array}\right]^{\mathrm{T}}, \boldsymbol{n}_{\mathrm{p}}=\left[\begin{array}{ll}n_{11} & -n_{3}\end{array}\right]^{\mathrm{T}}$ and

$$
H_{\mathrm{p}}=\left[\begin{array}{cc}
\sqrt{P_{\mathrm{p}}} h_{1} & \sqrt{P_{\mathrm{s}}} h_{4} \\
-\sqrt{P_{\mathrm{p}}} g h_{3}^{*} h_{2} & \sqrt{P_{\mathrm{s}} / 2} h_{4}^{*}
\end{array}\right] .
$$

Multiplying the orthogonalization vector $w_{\mathrm{p}}=\left[h_{4}^{*} \sqrt{P_{\mathrm{s}} / 2}-\sqrt{P_{\mathrm{s}}} h_{4}\right]$ to $y_{\mathrm{p}}$ we obtain,

$$
w_{\mathrm{p}} y_{\mathrm{p}}=\left(\sqrt{\frac{P_{\mathrm{s}}}{2}} \sqrt{P_{\mathrm{p}}} h_{4}^{*} h_{1}+g \sqrt{P_{\mathrm{p}} P_{\mathrm{s}}} h_{2} h_{4} h_{3}^{*}\right) x_{\mathrm{p}}+\sqrt{\frac{P_{\mathrm{s}}}{2}} h_{4}^{*} n_{11}+\sqrt{P_{\mathrm{s}}} h_{4} n_{3} .
$$

It is clear that the secondary signal $x_{\mathrm{s}}$ has been completely removed. Thus, the signal received at PR experiences no interference from the secondary transmission. The channel estimate $h_{4}$ required at PR for the orthogonalization vector $\boldsymbol{w}_{\mathrm{p}}$ can be obtained from the pilot-aided channel estimation procedures detailed later in Sect. III.C. The instantaneous received $\mathrm{SNR}$ at $\mathrm{PR}$ after the two-phase transmission is given by

$$
\begin{aligned}
\mathrm{SNR}_{\mathrm{p}} & =\frac{\left|h_{4}^{*} \sqrt{P_{\mathrm{s}} / 2} \sqrt{P_{\mathrm{p}}} h_{1}+\sqrt{P_{\mathrm{p}} P_{\mathrm{s}}} g h_{2} h_{4} h_{3}^{*}\right|^{2}}{\mathrm{E}\left[\left|\sqrt{P_{\mathrm{s}} / 2} h_{4}^{*} n_{11}+\sqrt{P_{\mathrm{s}}} h_{4} n_{3}\right|^{2}\right]} \\
& =\frac{P_{\mathrm{p}}\left\{\gamma_{1}+2 g^{2} \gamma_{2} \gamma_{3}+2 \sqrt{2} g R e\left(h_{2} h_{3}^{*} h_{1}\right)\right\}}{\left(2 g^{2} \gamma_{3}+3\right) \sigma^{2}} .
\end{aligned}
$$

The average received SNR at PR for the primary transmission can be derived as

$$
\begin{aligned}
\overline{\mathrm{SNR}}_{\mathrm{p}}= & \mathrm{E}\left[\mathrm{SNR}_{\mathrm{p}}\right] \\
= & \frac{d_{3}^{v} P_{\mathrm{p}}^{2}\left\{3 d_{3}^{v} P_{\mathrm{p}}-d_{2}^{v} P_{\mathrm{s}}-d_{2}^{v} P_{\mathrm{s}}\left[\ln \left(\frac{3 d_{3}^{v} P_{\mathrm{p}}}{d_{2}^{v} P_{\mathrm{s}}}\right)\right]\right\}}{d_{1}^{v}\left(3 d_{3}^{v} P_{\mathrm{p}}-d_{2}^{v} P_{\mathrm{s}}\right)^{2} \sigma^{2}} \\
& +\frac{P_{\mathrm{p}} P_{\mathrm{s}}\left\{9 d_{3}^{v 2} P_{\mathrm{p}}^{2}-P_{\mathrm{s}}^{2}\left(d_{2}^{v}\right)^{2}-6 d_{3}^{v} P_{\mathrm{p}} P_{\mathrm{s}} d_{2}^{v}\left[\ln \left(\frac{3 d_{3}^{v} P_{\mathrm{p}}}{d_{2}^{v} P_{\mathrm{s}}}\right)\right]\right\}}{\left(3 d_{3}^{v} P_{\mathrm{p}}-P_{\mathrm{s}} d_{2}^{v}\right)^{3} \sigma^{2}} .
\end{aligned}
$$

Please refer to Appendix A for the derivation. 
Average received SNR of secondary system with OSSS

1) Phase 1: In the 1st transmission phase, the signal received at SR is $\gamma_{\mathrm{sr}}^{(1)}$ which is given in (4).

2) Phase 2: The signal received at $S R$ in the 2 nd phase is

$$
y_{\mathrm{sr}}^{(2)}=\boldsymbol{h}_{\mathrm{s}} \boldsymbol{z}_{\mathrm{s}}+n_{22}
$$

where $\boldsymbol{h}_{\mathrm{s}}=\left[\begin{array}{ll}h_{5} & h_{6}\end{array}\right]$ and $n_{22} \sim \mathcal{C N}\left(0, \sigma^{2}\right)$ is the AWGN. Substituting (6) into (14) and taking the complex conjugate, we obtain

$$
\gamma_{\mathrm{sr}}^{(2) *}=\sqrt{\frac{P_{\mathrm{s}}}{2}} h_{6}^{*} x_{\mathrm{s}}-g \sqrt{P_{\mathrm{p}}} h_{5}^{*} h_{2} x_{\mathrm{p}}-n_{4} .
$$

where $n_{4}=g h_{5}^{*} n_{13}-n_{22}^{*}$. Thus, the signal at SR after the two-phase transmission can be written as

$$
y_{\mathrm{s}}=H_{\mathrm{s}} x+n_{\mathrm{s}}
$$

where $\gamma_{\mathrm{s}}=\left[\begin{array}{ll}y_{\mathrm{sr}}^{(1)} & \gamma_{\mathrm{sr}}^{(2) *}\end{array}\right]^{\mathrm{T}}, \boldsymbol{n}_{\mathrm{s}}=\left[\begin{array}{ll}n_{12}-n_{4}\end{array}\right]^{\mathrm{T}}$ and

$$
H_{\mathrm{s}}=\left[\begin{array}{cc}
\sqrt{P_{\mathrm{p}}} h_{7} & \sqrt{P_{\mathrm{s}}} h_{6} \\
-\sqrt{P_{\mathrm{p}}} g h_{5}^{*} & \sqrt{P_{\mathrm{s}} / 2} h_{6}^{*}
\end{array}\right] \text {. }
$$

Multiplying $\boldsymbol{y}_{\mathrm{s}}$ with an orthogonalization vector $\boldsymbol{w}_{\mathrm{s}}=\left[\sqrt{P_{\mathrm{p}}} h_{5}^{*} h_{2} \sqrt{P_{\mathrm{p}}} h_{7}\right]$, we obtain,

$$
w_{\mathrm{s}} y_{\mathrm{s}}=\left(\sqrt{\frac{P_{\mathrm{s}}}{2}} \sqrt{P_{\mathrm{p}}} h_{6}^{*} h_{7}+g \sqrt{P_{\mathrm{p}} P_{\mathrm{s}}} h_{6} h_{5}^{*} h_{2}\right) x_{\mathrm{s}}+g \sqrt{P_{\mathrm{p}}} h_{5}^{*} h_{2} h_{12}-\sqrt{P_{\mathrm{p}}} h_{7} n_{4} .
$$

It is clear from (18) that the primary signal $x_{\mathrm{p}}$ has been completely removed. Therefore, SR does not experience any interference from the primary transmission. The channel estimate $h_{7}$ and $h_{5}^{*} h_{2}$ required at SR for the orthogonalization vector $\boldsymbol{w}_{\mathrm{s}}$ can be obtained from the pilot-aided channel estimation procedures detailed in Sect. III.C. The instantaneous received SNR at SR after the two-phase transmission can be obtained as

$$
\begin{aligned}
\mathrm{SNR}_{\mathrm{s}} & =\frac{\left|h_{6}^{*} \sqrt{P_{\mathrm{s}} / 2} \sqrt{P_{\mathrm{p}}} h_{7}+g \sqrt{P_{\mathrm{p}} P_{\mathrm{s}}} h_{6} h_{5}^{*} h_{2}\right|^{2}}{E\left[\left|g \sqrt{P_{\mathrm{p}}} h_{5}^{*} h_{2} n_{12}-\sqrt{P_{\mathrm{p}}} h_{7} h_{4}\right|^{2}\right]} \\
& =\frac{P_{\mathrm{s}}\left(\gamma_{6} \gamma_{7}+2 g^{2} \gamma_{6} \gamma_{5} \gamma_{2}+2 \sqrt{2} g R e\left(h_{2} h_{5}^{*} h_{7}\right) \gamma_{6}\right)}{\left(g^{2} \gamma_{5} \gamma_{2}+g^{2} \gamma_{5} \gamma_{7}+\gamma_{7}\right) 2 \sigma^{2}} .
\end{aligned}
$$

The average received SNR at $\mathrm{SR}, \overline{\mathrm{SNR}}_{\mathrm{s}}$ is intractable and we will analyze it numerically.

\section{Channel estimation and other requirements}

For the various transmitting and receiving terminals in OSSS, we assume that channel estimation can be done through the pilot symbols in the control frames (CRTS, CCTS, and $\mathrm{CCTH}$ ) and data frames originating from PT and ST. With the help of pilot symbols in the CRTS frame, SR is able to estimate $h_{7}$. Similarly, $h_{4}$ can be obtained by PR by making use of the pilot symbols in CCTH. The product channel for PT-ST(1)-SR (the relay channel from PT to SR), i.e., $h_{2} h_{5}^{*}$ can be estimated at SR in the 2nd phase from PT's pilot symbols since ST (1) is an AF relay [34]. The multiplication of the orthogonalization vector at PR is similar to STBC decoding and thus we presume that the primary system supports STBC. Moreover, the flag indicating the switch from conventional decoding to STBC decoding at PR can be incorporated in $\mathrm{CCTH}$.

\section{Average received SNR for AF with superposition coding}

In this section we discuss and derive the average SNR for AF-SC protocol. The control signalling involved is exactly the same as OSSS which is given in Section IIB.

\section{Average received SNR of primary system with AF-SC}

1) Phase 1: The system model for the 1st transmission phase of AF-SC is shown in Figure 3. In this phase, both $\mathrm{ST}(1)$ and $\mathrm{ST}(2)$ overhears the signal transmitted from $\mathrm{PT}^{5}$. The channel coefficient

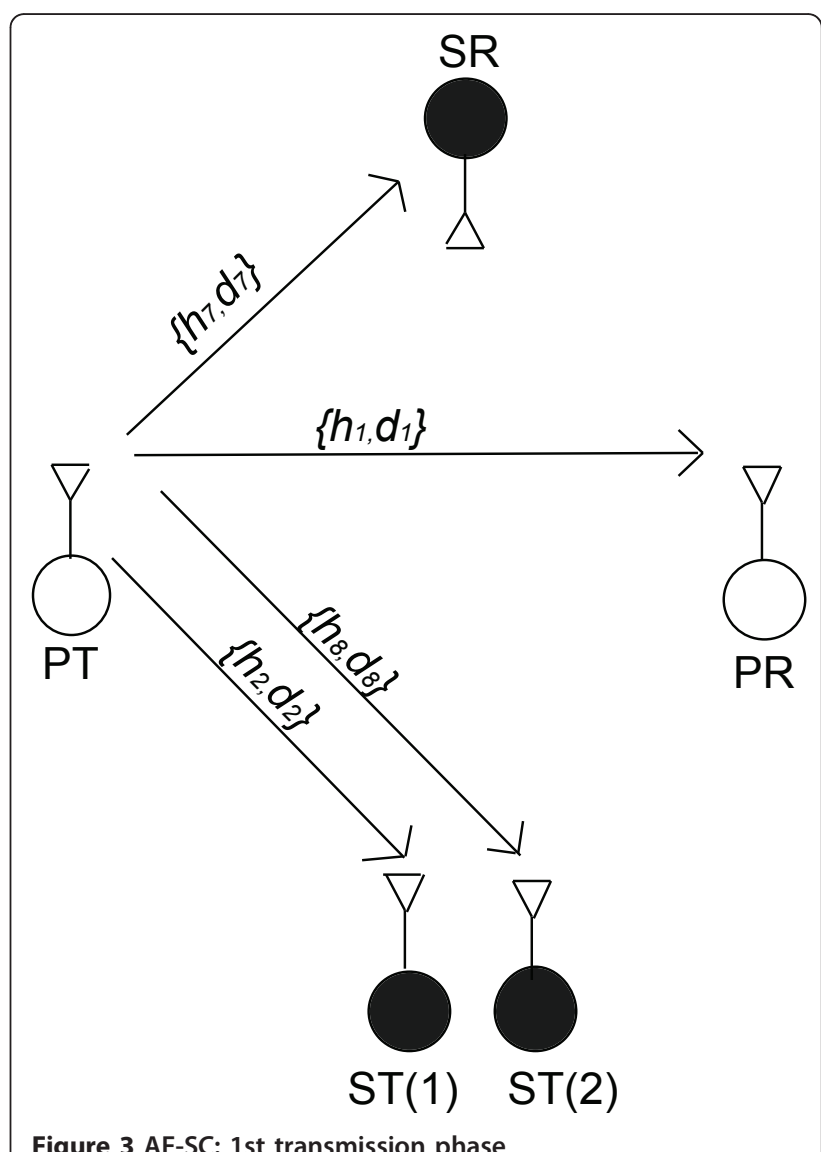


between PT-ST(2) is denoted by $h_{8}$ where $h_{8} \sim \mathcal{C N}\left(0, d_{8}^{-v}\right)$ and $\gamma_{8}=\left|h_{8}\right|^{2}$. Denoting the signals received by PR, ST and SR as $s_{\mathrm{pr}}^{(1)}, \boldsymbol{s}_{\mathrm{st}}$, and $s_{\mathrm{sr}}^{(1)}$, respectively, we have

$$
\begin{aligned}
& s_{\mathrm{pr}}^{(1)}=\sqrt{P_{\mathrm{p}}} h_{1} x_{\mathrm{p}}+\eta_{11}, \\
& \boldsymbol{s}_{\mathrm{st}}=\sqrt{P_{\mathrm{p}}}\left[\begin{array}{l}
h_{2} \\
h_{8}
\end{array}\right] x_{\mathrm{p}}+\left[\begin{array}{l}
\eta_{2} \\
\eta_{8}
\end{array}\right], \\
& s_{\mathrm{sr}}^{(1)}=\sqrt{P_{\mathrm{p}}} h_{7} x_{\mathrm{p}}+\eta_{14},
\end{aligned}
$$

where $s_{\mathrm{st}}=\left[s_{\mathrm{st}}^{(2)} s_{\mathrm{st}}^{(8)}\right]^{\mathrm{T}} \cdot s_{\mathrm{st}}^{(2)}$ and $s_{\mathrm{st}}^{(8)}$ are the signal received by $\mathrm{ST}(1)$ and $\mathrm{ST}(2)$, respectively, and $\eta_{11}, \eta_{2}$, $\eta_{8}, \eta_{14}$ are the AWGN with variance $\sigma^{2}$ at the respective receivers. ST will then select the received signal with a higher received power, i.e., $s_{\mathrm{st}}^{\left(\tau_{\mathrm{opt}}\right)}=\sqrt{P_{\mathrm{p}}} h_{\tau_{\mathrm{opt}}} x_{\mathrm{p}}+\eta_{\tau_{\mathrm{opt}}} \tau_{\mathrm{opt}} \in\{2,8\}$ where

$$
\tau_{\mathrm{opt}}=\arg \max _{\tau \in\{2,8\}}\left(\left|s_{\mathrm{st}}^{(\tau)}\right|^{2}\right) .
$$

As a result, selection diversity is achieved at $\mathbf{S T}$ in the 1st phase. After performing selection, ST normalizes the received primary signal based on its power constraint and further amplifies it with the power allocation factor $\alpha$ where $0 \leq \alpha \leq 1$. The remaining power $(1-\alpha)$ is assigned to the secondary signal. Thus, the signal vector regenerated from $\mathbf{S T}$ can be written as

$$
v_{\mathrm{st}}=V x_{\mathrm{a} f}
$$

where $v_{\mathrm{st}}=\left[v_{\mathrm{st}}^{(1)} v_{\mathrm{st}}^{(2)}\right]^{\mathrm{T}}$ is the transmit vector from $\mathbf{S T}$, and $v_{\mathrm{st}}^{(1)}, v_{\mathrm{st}}^{(2)}$ are the signals from $\mathrm{ST}(1)$ and $\mathrm{ST}(2)$, respectively, ${ }^{6}$

$$
V=\left[\begin{array}{cc}
\kappa \sqrt{\alpha} & 0 \\
0 & \sqrt{(1-\alpha) P_{\mathrm{s}}}
\end{array}\right]
$$

$x_{\mathrm{a} f}=\left[s_{\mathrm{st}}^{\left(\tau_{\mathrm{opt}}\right)} x_{\mathrm{s}}\right]^{\mathrm{T}}$ and the power normalization factor is given by $\kappa=\sqrt{\frac{P s}{\left(P_{\mathrm{p}} \gamma_{\tau_{\mathrm{opt}}}+\sigma^{2}\right)}}$.

2) Phase 2: The system model for the 2nd transmission phase of AF-SC is the same as OSSS as shown in Figure 2. In this phase, the signal received by PR is given by

$$
\boldsymbol{s}_{\mathrm{pr}}^{(2)}=\boldsymbol{h}_{\mathrm{af}} \boldsymbol{v}_{\mathrm{st}}+\eta_{21}
$$

where $\boldsymbol{h}_{a f}=\left[\begin{array}{ll}h_{3} & h_{4}\end{array}\right]$ and $\eta_{21} \sim \mathcal{C N}\left(0, \sigma^{2}\right)$ is the AWGN. After substituting (24) in (26) we obtain,

$$
s_{\mathrm{pr}}^{(2)}=\left(\sqrt{P_{\mathrm{p}} \alpha} \kappa h_{\tau_{\mathrm{opt}}} h_{3}\right) x_{\mathrm{p}}+\left(\sqrt{P_{\mathrm{s}}(1-\alpha)} h_{4}\right) x_{\mathrm{s}}+\sqrt{\alpha} \kappa h_{3} \eta_{\tau_{\mathrm{opt}}}+\eta_{21} .
$$

Unlike OSSS, $s_{\mathrm{pr}}^{(2)}$ also contains interference from the secondary signal. This interference limits the achievable performance of primary system in AF-SC. The signals $s_{\mathrm{pr}}^{(1)}$ and $s_{\mathrm{pr}}^{(2)}$ are then combined at PR using MRC for decoding of $x_{\mathrm{p}}$. The SNR after MRC is given by

$$
\mathrm{SNR}_{\mathrm{P}}^{\mathrm{AF}-\mathrm{SC}}=\frac{P_{\mathrm{p}} \gamma_{1}}{\sigma^{2}}+\frac{P_{\mathrm{p}} \gamma_{\tau_{\mathrm{opt}}} \gamma_{3} \kappa^{2} \alpha}{P_{\mathrm{S}}(1-\alpha) \gamma_{4}+\alpha \kappa^{2} \gamma_{3} \sigma^{2}+\sigma^{2}}
$$

The average received SNR at PR, PR, $\overline{\mathrm{SNR}}_{\mathrm{p}}^{\mathrm{AF}-\mathrm{SC}}$ for AF-SC is intractable and we will analyze it numerically.

\section{Average received SNR of secondary system with AF-SC}

1) Phase 1: The signal received at $S R$ in the $1 \mathrm{st}$ transmission phase is given by

$$
s_{\mathrm{sr}}^{(1)}=\sqrt{P_{\mathrm{p}}} h_{7} x_{\mathrm{p}}+\eta_{13}
$$

where $\eta_{13} \sim \mathcal{C N}\left(0, \sigma^{2}\right)$ is the AWGN. At SR, an estimate of $x_{\mathrm{p}}$ is obtained using (29) as

$$
\widehat{x_{\mathrm{p}}}=\frac{s_{\mathrm{sr}}^{(1)}}{\sqrt{P_{\mathrm{p}}} h_{7}}=x_{\mathrm{p}}+\frac{\eta_{13}}{\sqrt{P_{\mathrm{p}}} h_{7}} \text {. }
$$

2) Phase 2: The signal received at $S R$ in the 2 nd transmission phase is

$$
\boldsymbol{s}_{\mathrm{sr}}^{(2)}=\boldsymbol{h}_{a f}^{\mathrm{s}} \boldsymbol{v}_{\mathrm{st}}+\eta_{22}
$$

where $\boldsymbol{h}_{\text {af }}^{\mathrm{s}}=\left[h_{5} h_{6}\right]$. and $\eta_{22} \sim \mathcal{C N}\left(0, \sigma^{2}\right)$ is the AWGN. Substituting (24) in (31) we obtain

$$
s_{\mathrm{sr}}^{(2)}=\left(\sqrt{P_{\mathrm{p}} \alpha} \kappa h_{\tau_{\mathrm{opt}}} h_{5}\right) x_{\mathrm{p}}+\left(\sqrt{P_{\mathrm{s}}(1-\alpha)} h_{6}\right) x_{\mathrm{s}}+\sqrt{\alpha} \kappa h_{5} \eta_{\tau_{\mathrm{opt}}}+\eta_{22} .
$$

The estimate $\widehat{x_{\mathrm{p}}}$ in (30) is used to cancel out the interference component $\left(\sqrt{P_{\mathrm{p}} \alpha} \kappa h_{\tau_{\mathrm{opt}}} h_{5}\right) x_{\mathrm{p}}$ from $s_{\mathrm{sr}}^{(2)}$, to obtain

$$
s_{\mathrm{sr}}^{\widehat{(2)}}=\left(\sqrt{P_{\mathrm{s}}(1-\alpha)} h_{6}\right) x_{\mathrm{s}}-\frac{\sqrt{\alpha} \kappa h_{\tau_{\mathrm{opt}}} h_{5} \eta_{13}}{h_{7}}+\sqrt{\alpha} \kappa h_{5} \eta_{\tau_{\mathrm{opt}}}+\eta_{22}
$$

The channel estimate $h_{\tau_{\mathrm{opt}}} h_{5}$ and $h_{\tau_{\mathrm{opt}}}$ required at SR for interference cancellation can be obtained through pilot-aided channel estimation procedures detailed in Sect. III.C and [35]. Therefore, the SNR at SR can be obtained as 


$$
\mathrm{SNR}_{\mathrm{s}}^{\mathrm{AF}-\mathrm{SC}}=\frac{P_{\mathrm{s}}(1-\alpha) \gamma_{6} \gamma_{7}}{\alpha \kappa^{2}\left(\gamma_{\tau_{\mathrm{opt}}}+\gamma_{7}\right) \gamma_{5} \sigma^{2}+\gamma_{7} \sigma^{2}}
$$

The average received SNR at $\mathrm{SR}, \overline{\mathrm{SNR}}_{\mathrm{S}}^{\mathrm{AF}-\mathrm{SC}}$ is intractable and we will analyze it numerically.

\section{Simulation results and discussion}

For ease of exposition, PT, SR, ST and PR are assumed to be collinear and the distance between ST(1) and ST (2) is assumed to be much smaller than the distance between the other system nodes, thus $d_{2} \approx d_{8}, d_{3} \approx d_{4}$ and $d_{5} \approx d_{6}$. The position of PT, SR, ST and PR are fixed to $(0,0),(0.25,0),(0.5,0)$ and $(1,0)$, respectively, as shown in Figure 4. The path loss component is chosen to be $v=4$. Thus all the radio links between PT, PR, ST and SR can be characterized by their respective positions on the straight line.

Figure 5 shows the average SNR performance of primary system for OSSS, $\overline{\mathrm{SNR}}_{\mathrm{p}}$ with respect to $\frac{P_{\mathrm{s}}}{\sigma^{2}}$ for different values of $\frac{P_{\mathrm{p}}}{\sigma^{2}}$. The corresponding plot for secondary system, $\overline{\mathrm{SNR}}_{\mathrm{s}}$, is shown in Figure 6. For comparison purposes, we have also plotted the results for $\overline{\mathrm{SNR}}_{\mathrm{MRC}}$ which is the average received SNR of primary system for direct transmission with ARQ. $\overline{\mathrm{SNR}}_{\mathrm{MRC}}$ will be a useful benchmark for comparison as $\overline{\mathrm{SNR}}_{\mathrm{MRC}}$ shows the performance of primary system with retransmission in the absence of any secondary system. Good agreement between the simulation and theoretical results for $\overline{\mathrm{SNR}}_{\mathrm{p}}$ and $\overline{\mathrm{SNR}}_{\mathrm{MRC}}$ in Figure 5 validates the analytical results obtained in this article.

From Figures 5 and 6, it can be observed that the performance of primary as well as secondary system for OSSS improves with an increase in $\frac{P_{\mathrm{s}}}{\sigma^{2}}$ for a given value of $\frac{P_{\mathrm{p}}}{\sigma^{2}}$. This proves that the secondary transmission does not interfere with the primary transmission; in fact it contributes to the performance of the primary transmission. Moreover, it also shows that an increase in secondary transmission power $P_{\mathrm{s}}$ benefits both the primary as well as secondary systems. Another observation that can be made from Figure 5 is that when the primary system is interested in improving its QoS (e.g., $\overline{\mathrm{SNR}}_{\mathrm{T}}>13 \mathrm{~dB}$ at
$\frac{P_{\mathrm{p}}}{\sigma^{2}}=20 \mathrm{~dB}$ or $\overline{\mathrm{SNR}}_{\mathrm{T}}>23 \mathrm{~dB}$ at $\left.\frac{P_{\mathrm{p}}}{\sigma^{2}}=20 \mathrm{~dB}\right)$, it can always request the help of $\mathbf{S T}$ to improve its QoS while at the same time allowing spectrum access by the secondary system. QoS improvement of up to $8 \mathrm{~dB}$ can be achieved by the primary system in the case of OSSS with respect to $\overline{\mathrm{SNR}}_{\mathrm{MRC}}$ at $\frac{P_{\mathrm{s}}}{\sigma^{2}}=40 \mathrm{~dB}$ for both $\frac{P_{\mathrm{p}}}{\sigma^{2}}=10 \mathrm{~dB}$ and $\frac{P_{\mathrm{p}}}{\sigma^{2}}=20 \mathrm{~dB}$. From Figure 5, we can also conclude that if QoS requirement for the primary system is set too high (e.g., $\overline{\mathrm{SNR}}_{\mathrm{T}}>22 \mathrm{~dB}$ at $\frac{P_{\mathrm{p}}}{\sigma^{2}}=10 \mathrm{~dB}$ ), $\overline{\mathrm{SNR}}_{\mathrm{p}}<\overline{\mathrm{SNR}}_{\mathrm{T}}$ and secondary spectrum access is not possible. This limitation is due to the noise amplification at $\mathrm{ST}(1)$ in the AF relaying. Thus when $\overline{\mathrm{SNR}}_{\mathrm{T}}$ requirement is reasonable, secondary system is always able to achieve spectrum access as long as it is willing to increase its transmit power such that $\overline{\mathrm{SNR}}_{\mathrm{T}}$ is met.

Figures 5 and 6, respectively, show $\overline{\mathrm{SNR}}_{\mathrm{p}}^{\mathrm{AF}-\mathrm{SC}}$ and $\overline{\mathrm{SNR}}_{\mathrm{S}}^{\mathrm{AF}-\mathrm{SC}}$ for AF-SC at $\alpha=0.5$ and $\alpha=0.9$. From the two figures it can be easily deduced that there is a trade-off between the performance of primary and secondary systems, and the performance of one system is limited by the interference from the other system. As we increase the value of $\alpha$, the performance of primary system improves whereas the performance of secondary system deteriorates and vice versa. In AF-SC, the performance of primary system is limited by the interference from the secondary system as well as amplified noise in the 2nd phase. From Figure 5, at $\frac{P_{\mathrm{p}}}{\sigma^{2}}=20 \mathrm{~dB} \overline{\mathrm{SNR}}_{\mathrm{p}}^{\mathrm{AF}-\mathrm{SC}}<\overline{\mathrm{SNR}}_{\mathrm{MRC}}$ for all values of $\frac{P_{\mathrm{s}}}{\sigma^{2}}$ even with $\alpha=0.9$. Thus there is no possibility of spectrum access for the secondary system in this case. Furthermore, for $\alpha=0.9$ at $\frac{P_{\mathrm{p}}}{\sigma^{2}}=10 \mathrm{~dB}$, AF-SC achieves the closest possible performance to OSSS for the primary system, but OSSS outperforms AF-SC by a large margin for the secondary transmission as can be observed from Figure 6.

\section{Conclusions}

In this article, we proposed a two-phase OSSS based on cooperative amplify-and-forward relaying for a WSN (a.k.a secondary system) to achieve spectrum

$$
\text { PT }(0,0) \quad \text { SR }(0.25,0) \quad \text { ST }(0.5,0) \quad \text { PR }(1,0)
$$

Figure 4 System configuration for simulation. 


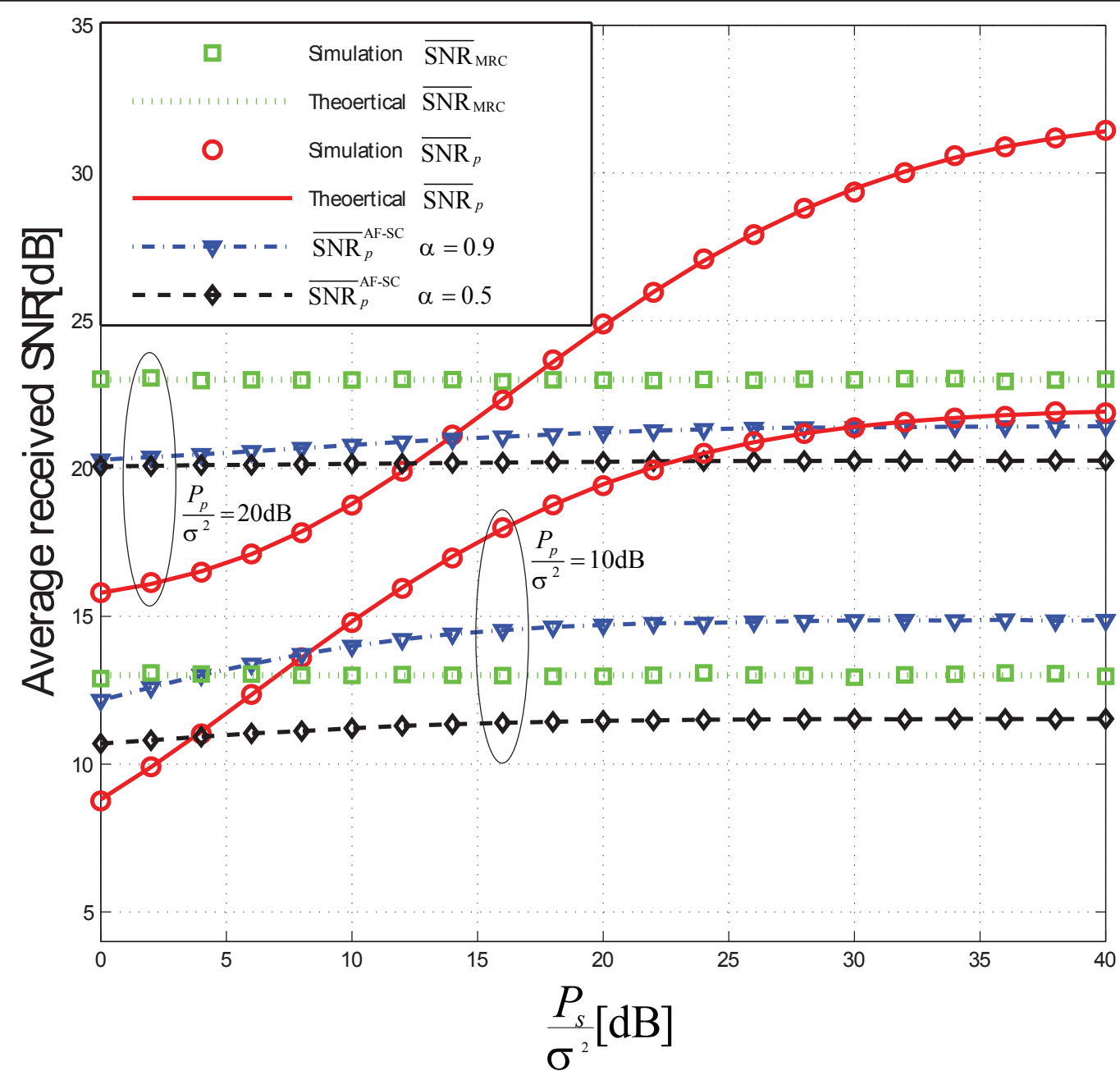

Figure 5 Average received SNR of primary transmission for various values of $\frac{p_{\mathrm{s}}}{\sigma^{2}}$ for OSSS, AF-SC, and direct transmission with ARQ. Theoretical and simulation values are reported for $\overline{\mathrm{SNR}}_{\mathrm{p}}$ and $\overline{\mathrm{SNR}}_{\mathrm{MRC}}$, whereas only simulation values are reported for $\overline{\mathrm{SNR}}_{\mathrm{p}}^{\mathrm{AF}-\mathrm{SC}}$.

access along with a primary system. We showed that by using the proposed scheme, the two systems can co-exist in the same frequency band without causing any interference to one another. Moreover, when the PT-PR link is weak, WSN can be used to enhance the QoS of the primary system. We further showed that in OSSS, WSN is always able to achieve spectrum access as long as it is willing to increase its transmit power such that $\overline{\mathrm{SNR}}_{\mathrm{T}}$ is met.

We analyzed the performance of OSSS by obtaining closed form expressions for the average SNR of the primary system. In order to validate its efficiency, we also analyzed an interference limited scheme (AF-SC) and compared it with OSSS. Simulation results showed that performance of OSSS is always better than AF-SC for both the primary system and WSN.

\section{Appendix A Derivation for average SNR of primary system with OSSS}

From (12) and (13), we obtain

$$
\begin{aligned}
\overline{\mathrm{SNR}}_{\mathrm{p}} & =\frac{P_{\mathrm{p}}}{\sigma^{2}} \mathrm{E}\left[\frac{\gamma_{1}+2 g^{2} \gamma_{2} \gamma_{3}+2 \sqrt{2} \operatorname{Re}\left(h_{2} h_{3}^{*} h_{1}\right) g}{\left(2 g^{2} \gamma_{3}+3\right)}\right] \\
& =\frac{P_{\mathrm{p}}}{\sigma^{2}}\left(\delta_{1}+\delta_{2}+\delta_{3}\right)
\end{aligned}
$$

where $\delta_{1}=\mathrm{E}\left[\frac{\gamma 1}{2 g^{2} \gamma_{3}+3}\right], \quad \delta_{2}=\mathrm{E}\left[\frac{2 g^{2} \gamma_{2} \gamma_{3}}{2 g^{2} \gamma_{3}+3}\right] \quad$ and $\delta_{3}=\mathrm{E}\left[\frac{2 \sqrt{2} g \operatorname{Re}\left(h_{3}^{*} h_{1} h_{2}\right)}{2 g^{2} \gamma_{3}+3}\right] \cdot \delta_{1}$ can be evaluated as

$$
\delta_{1}=\int_{0}^{\infty} \int_{0}^{\infty} \int_{0}^{\infty}\left(\frac{\gamma_{1}}{2 g^{2} \gamma_{3}+3}\right) p_{\gamma_{1}}\left(\gamma_{1}\right) p_{\gamma_{2}}\left(\gamma_{2}\right) p_{\gamma_{3}}\left(\gamma_{3}\right) d \gamma_{1} d \gamma_{2} d \gamma_{3}
$$




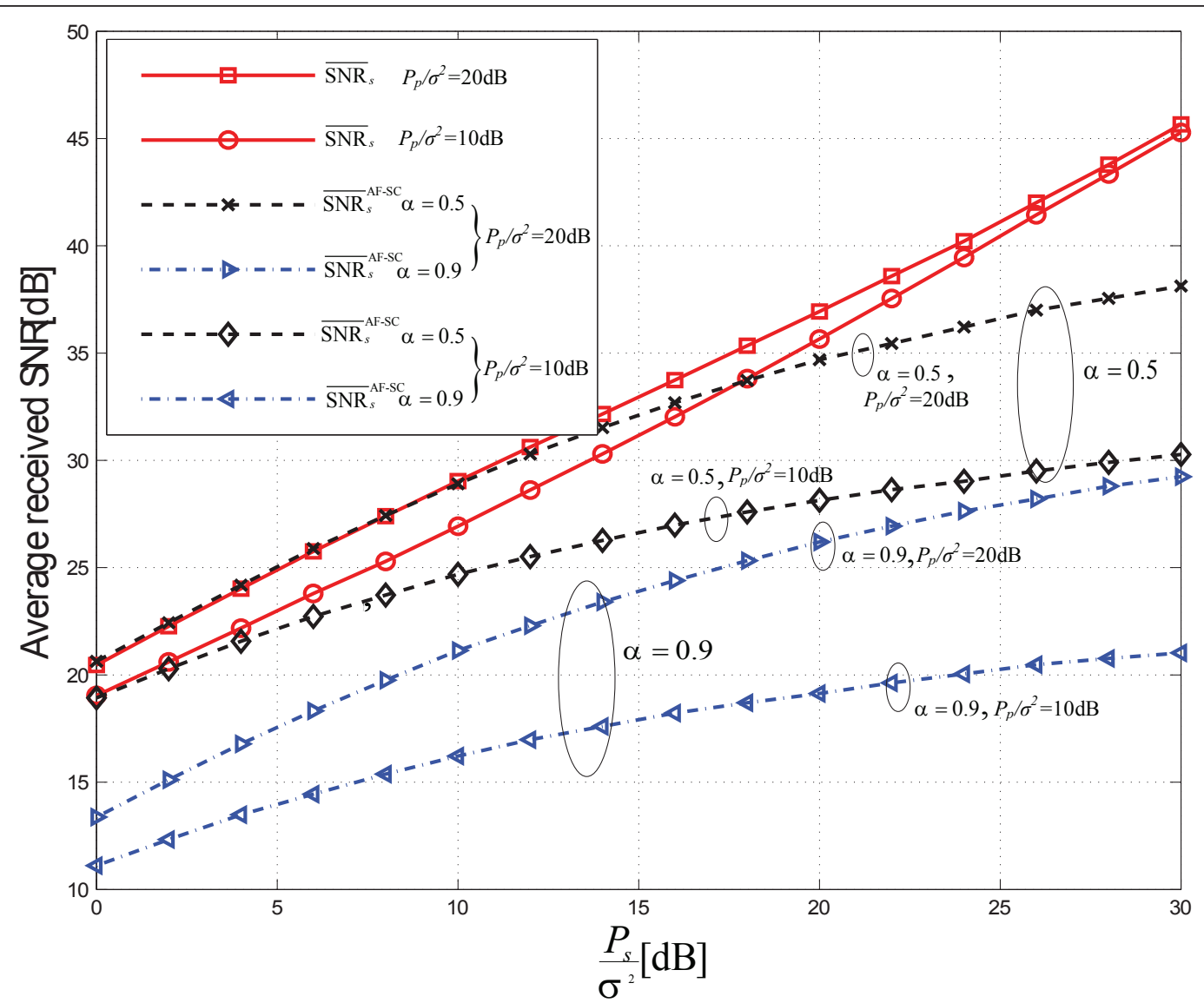

Figure 6 Average received SNR of secondary transmission for various values of $\frac{p_{\mathrm{s}}}{\sigma^{2}}$ for OSSS and AF-SC.

where $p_{\gamma_{1}}\left(\gamma_{1}\right), p_{\gamma_{2}}\left(\gamma_{2}\right)$ and $p_{\gamma_{3}}\left(\gamma_{3}\right)$ are the probability density function (pdf) of $\gamma_{1}, \gamma_{2}$ and $\gamma_{3}$, respectively. Additionally, $\gamma_{i} \sim \varepsilon\left(d_{i}^{v}\right), i=1,2$, 3. Thus from (36),

$$
\begin{aligned}
\delta_{1} & =\int_{0}^{\infty} \int_{0}^{\infty} \frac{\gamma_{1}}{2 g^{2} \gamma_{3}+3} p_{\gamma_{2}}\left(\gamma_{2}\right) p_{\gamma_{3}}\left(\gamma_{3}\right) d \gamma_{2} d \gamma_{3} \int_{0}^{\infty} \gamma_{1} p_{\gamma_{1}}\left(\gamma_{1}\right) d \gamma_{1} \\
& =\frac{1}{d_{1}^{v}} \int_{0}^{\infty} \int_{0}^{\infty} \frac{\gamma_{1}}{2 g^{2} \gamma_{3}+3} p_{\gamma_{2}}\left(\gamma_{2}\right) p_{\gamma_{3}}\left(\gamma_{3}\right) d \gamma_{2} d \gamma_{3} \\
& =\frac{1}{d_{1}^{v}} \int_{0}^{\infty} p_{\gamma_{3}}\left(\gamma_{3}\right) \int_{0}^{\infty} p_{\gamma_{2}}\left(\gamma_{2}\right) \frac{1}{\frac{P_{\mathrm{s}}}{P_{\mathrm{p}} \gamma_{2}+\sigma^{2}} \gamma_{3}+3} d \gamma_{2} d \gamma_{3} .
\end{aligned}
$$

Assuming $\frac{\sigma^{2}}{P_{\mathrm{p}}} \approx 0$, then (37) can be rewritten as

$$
\begin{aligned}
& \delta_{1} \approx \frac{1}{d_{1}^{v}} \int_{0}^{\infty} p_{\gamma_{3}}\left(\gamma_{3}\right) \int_{0}^{\infty} p_{\gamma_{2}}\left(\gamma_{2}\right) \frac{1}{\frac{P_{s}}{P_{\mathrm{p}} \gamma_{2}} \gamma_{3}+3} d \gamma_{2} d \gamma_{3} \\
& =d_{3}^{v} P_{\mathrm{p}}\left(-d_{2}^{v} P_{\mathrm{s}}+3 d_{3}^{v} P_{\mathrm{p}}-d_{2}^{v} P_{\mathrm{s}} \ln (3)+d_{2}^{v} P_{\mathrm{s}} \ln \left(d_{2}^{v}\right)+d_{2}^{v} P_{\mathrm{s}} \ln \left(P_{\mathrm{s}}\right)-d_{2}^{v} P_{\mathrm{s}} \ln \left(d_{3}^{v}\right)-d_{2}^{v} P_{\mathrm{s}} \ln \left(P_{\mathrm{p}}\right)\right) \\
& =d_{3}^{v} \frac{P_{\mathrm{p}}\left\{-d_{2}^{v} P_{\mathrm{s}}+3 d_{3}^{v} P_{\mathrm{p}}-d_{2}^{v} P_{\mathrm{s}}\left[\ln \left(\frac{3 d_{3} P_{\mathrm{p}}}{d_{2}^{v} P_{\mathrm{s}}}\right)\right]\right\}}{d_{1}^{v}\left(-d_{2}^{v} P_{\mathrm{s}}+3 d_{3}^{v} P_{\mathrm{p}}\right)^{2}}
\end{aligned}
$$

Similarly, we can obtain

$$
\begin{aligned}
\delta_{2} & =\int_{0}^{\infty} \int_{0}^{\infty}\left(\frac{2 g^{2} \gamma_{2} \gamma_{3}}{2 g^{2} \gamma_{3}+3}\right) p_{\gamma_{2}}\left(\gamma_{2}\right) p_{\gamma_{3}}\left(\gamma_{3}\right) d \gamma_{2} d \gamma_{3} \\
& \approx \int_{0}^{\infty} \int_{0}^{\infty} p_{\gamma_{3}}\left(\gamma_{3}\right) \int_{0}^{\infty} p_{\gamma_{2}}\left(\gamma_{2}\right) \frac{\frac{P_{\mathrm{s}}}{P_{\mathrm{p}} \gamma_{2}} \gamma_{2} \gamma_{3}}{\frac{P_{\mathrm{s}}}{P_{\mathrm{p}} \gamma_{2}} \gamma_{3}+3} d \gamma_{2} d \gamma_{3} \\
& =\frac{P_{\mathrm{s}}\left\{-P_{\mathrm{s}}^{2}\left(d_{2}^{\nu}\right)^{2}+9 d_{3}^{\nu 2} P_{\mathrm{p}}^{2}-6 d_{3}^{v} P_{\mathrm{p}} P_{\mathrm{s}} d_{2}^{v}\left[\ln \left(\frac{3 d_{3}^{v} P_{\mathrm{p}}}{d_{2}^{\nu} P_{\mathrm{s}}}\right)\right]\right\}}{\left(-P_{\mathrm{s}} d_{2}^{v}+3 d_{3}^{v} P_{\mathrm{p}}\right)^{3}}
\end{aligned}
$$

and $\delta_{3}=0$. Thus substituting (38) and (39) in (35) we obtain

$$
\begin{aligned}
\overline{\mathrm{SNR}}_{\mathrm{p}}= & \frac{d_{3}^{v} P_{\mathrm{p}}^{2}\left\{3 d_{3}^{v} P_{\mathrm{p}}-d_{2}^{v} P_{\mathrm{s}}-d_{2}^{v} P_{\mathrm{s}}\left[\ln \left(\frac{3 d_{3}^{v} P_{\mathrm{p}}}{d_{2}^{v} P_{\mathrm{s}}}\right)\right]\right\}}{d_{1}^{v}\left(3 d_{3}^{v} P_{\mathrm{p}}-d_{2}^{v} P_{\mathrm{s}}\right)^{2} \sigma^{2}} \\
& +\frac{P_{\mathrm{p}} P_{\mathrm{s}}\left\{9 d_{3}^{v 2} P_{\mathrm{p}}^{2}-P_{\mathrm{s}}^{2}\left(d_{2}^{v}\right)^{2}-6 d_{3}^{v} P_{\mathrm{p}} P_{\mathrm{s}} d_{2}^{v}\left[\ln \left(\frac{3 d_{3}^{v} P_{\mathrm{p}}}{d_{2}^{v} P_{\mathrm{s}}}\right)\right]\right\}}{\left(3 d_{3}^{v} P_{\mathrm{p}}-P_{\mathrm{s}} d_{2}^{v}\right)^{3} \sigma^{2}} .
\end{aligned}
$$




\section{End notes}

${ }^{1}$ http://www.ntia.doc.gov/osmhome/allochrt.pdf.

${ }^{2}$ It should be noted that whether $\mathbf{S T}$ is able to assist $\mathrm{PT}$ or not, is a probabilistic event due to the random fading channels.

${ }^{3}$ However, in return for an opportunity to access the spectrum, there will be an increase in hardware complexity and cost.

${ }^{4}$ Please note that ST(1) and ST(2) continuously update each other of the information that needs to be send to the SR. Thus, in the 1st phase, even if ST(1) receives the signal $x_{\mathrm{s}}$ from ST(2), it has a priori knowledge of $x_{\mathrm{s}}$ so it can be cancelled out easily from the received signal at $\mathrm{ST}(1)$.

${ }^{5}$ If there is only one $\mathrm{ST}$ node, then AF-SC reduces to the spectrum sharing scheme proposed in [35].

${ }^{6} \mathrm{We}$ may consider other choices such as $V=\left[\begin{array}{c}\kappa \sqrt{\frac{\alpha}{2}} \sqrt{\frac{(1-\alpha) P_{s}}{2}} \\ \kappa \sqrt{\frac{\alpha}{2}} \sqrt{\frac{(1-\alpha) P_{s}}{2}}\end{array}\right]$ or $V=\left[\begin{array}{cc}\kappa \sqrt{\alpha} & \sqrt{(1-\alpha) P_{s}} \\ 0 & 0\end{array}\right]$.

Though not given in this article, simulation results show that the $V$ we used in (25) achieves the best performance among the three.

\section{Abbreviations}

AF-SC: AF with superposition coding; AWGN: additive white Gaussian noise; CBS: central base station; CCTS: cooperative clear-to-send; CCTH: cooperative clear-to-help; CRTS: cooperative right-to-send; CSI: channel state information; DSA: dynamic spectrum access; MRC: maximum ratio combining; MEMS: microelectro-mechanical systems; NTIA: National Telecommunications and Information Administrations; OSSS: Orthogonal Spectrum Sharing Scheme; PT: primary transmitter; PR: primary receiver; QOS: Quality of Service; SR: secondary receiver; STs: Secondary transmitters; SNR: signal to noise ratio; STBC: space time block code; WSNs: wireless sensor networks.

\section{Acknowledgements}

This work is supported by the Singapore Ministry of Education Academic Research Fund Tier 2, MOE2009-T2-2-059.

\section{Author details}

'School of Electrical and Electronic Engineering, Nanyang Technological University, Singapore, 639801 Singapore ${ }^{2}$ Philips Research, High Tech Campus, 5656AE Eindhoven, The Netherlands

\section{Competing interests}

The authors declare that they have no competing interests.

Received: 23 December 2010 Accepted: 10 June 2011

Published: 10 June 2011

\section{References}

1. R Frank, Understanding Smart Sensors (Artech House, Norwood, MA, 2000)

2. IF Akyildiz, W Su, Y Sankarasubramaniam, E Cayirci, Wireless sensor networks: a survey. Int J Comput Telecommun Netw. 38(4), 393-422 (2002)

3. FL Lewis, Wireless sensor networks, in Smart Environments: Technology, Protocols, and Applications, ed. by Cook DJ, Das SK (Wiley, New York, 2004)

4. A Kansal, M Srivastava, Energy-harvesting-aware power management, in Wireless Sensor Networks, ed. by Bulusu N, Jha S (Artech House, Norwood, MA, 2005)
5. I Demirkol, C Ersoy, F Alagoz, MAC protocols for wireless sensor networks. IEEE Commun Mag. 44(4), 115-121 (2006)

6. G Zhou, JA Stankovic, S Son, Crowded spectrum in wireless sensor networks, in Proceedings of IEEE EmNets, (2006)

7. Q Zhao, L Tong, A Swami, Decentralized cognitive MAC for opportunistic spectrum access in ad hoc networks: a POMDP framework. IEEE J Select Areas Commun. 25, 224-232 (2007)

8. Federal Communications Commission, Spectrum policy task force, Rep. ET Docket no. 02-135, Nov 2002

9. Q Zhao, BM Sadler, A survey of dynamic spectrum access: signal processing, networking, and regulatory policy. IEEE Signal Process Mag. 24(3), 79-89 (2007)

10. IF Akyildiz, W-Y Lee, MC Vuran, S Mohanty, Next generation/dynamic spectrum access/cognitive radio wireless networks: a survey. Comput Netw. 50(13), 2127-2159 (2006)

11. Y Xing, R Chandramouli, S Mangold, S Shankar, Dynamic spectrum access in open spectrum wireless networks. IEEE J Select Areas Commun. 24(3), 626-637 (2006)

12. A Goldsmith, SA Jafar, I Maric, S Srinivasa, Breaking spectrum gridlock with cognitive radios: an information theoretic perspective. in Proceedings of IEEE. 97, 894-914 (2009)

13. Y Han, SH Ting, A Pandharipande, Cooperative decode-and-forward relaying for secondary spectrum access. IEEE Trans Wireless Commun. 8(10), 4945-4950 (2009)

14. Q Li, SH Ting, A Pandharipande, Y Han, Cognitive spectrum sharing with two-way relaying systems. IEEE Trans Veh Technol. 60(3), 1233-1240 (2011)

15. S Sriram, S Vishwanath, On the capacity of a class of MIMO cognitive radios. IEEE J Select Top Signal Process. 2(1), 103-117 (2008)

16. S Sriram, S Vishwanath, S Jafar, S Shamai, On the capacity of cognitive relay assisted Gaussian interference channel, in Proceedings of 2008 International Symposium On Information Theory, Toronto, Canada, July 2008

17. ECVD Meulen Three-terminal communication channels. Adv Appl Prob. 3, 120-154 (1971)

18. JN Laneman, DNC Tse, GW Wornell, Cooperative diversity in wireless networks: efficient protocols and outage behavior. IEEE Trans Inform Theory. 50, 3062-3080 (2004)

19. G Kramer, M Gastpar, P Gupta, Cooperative strategies and capacity theorems for relay networks. IEEE Trans Inform Theory, 51, 3037-3063 (2005)

20. VA Bohara, SH Ting, Preliminary measurement results for cognitive spectrum sharing based on cooperative relaying. in Proceedings of International International conference on wireless communication and signal processing, Suzhou, China, Oct. 2010.

21. H Wicaksana, SH Ting, CK Ho, WH Chin, YL Guan, AF two-path half duplex relaying with inter-relay self interference cancellation: diversity analysis and its improvement. IEEE Trans Wireless Commun. 8(9), 4720-4729 (2009)

22. C Fischione, K Johansson, F Graziosi, F Santucci, Distributed cooperative processing and control over wireless sensor networks. in Proceedings of the International Conference on Communications and Mobile Computing, 1311-1316 (2007)

23. H Yang, HY Shen, B Sikdar, A MAC protocol for cooperative MIMO transmissions in sensor networks. in Proceedings of IEEE Global Communications Conference, Exhibition and Industry Forum, Washington, USA, 636-640 (2007)

24. P Liu, Z Tao, S Narayanan, T Korakis, SS Panwar, CoopMAC: a cooperative MAC for wireless LANs. IEEE J Select Areas Commun. 25(2), 340-354 (2007)

25. A Bletsas, A Khisti, DP Reed, A Lippman, A simple cooperative diversity method based on network path selection. IEEE J Select Areas Commun. 24(3), 659-672 (2006)

26. A Sharma, V Gelara, S Singh, T Korakis, P Liu, S Panwar, Implementation of a cooperative MAC protocol using a software defined radio platform. in Proceedings of IEEE LANMAN, Cluj-Napoca, Romania, Sept. 2008

27. S Valentin, HS Lichte, H Karl, S Simoens, G Vivier, J Vidal, A Agustin, Implementing cooperative wireless networks. in Cognitive Wireless Networks, ed. by Fitzek FHP, Katz MD (Netherlands, Springer, 2007), pp. 155-178

28. Y Han, SH Ting, A Pandharipande, Cooperative spectrum sharing protocol with secondary user selection. IEEE Trans Wireless Commun. 9(9), 2914-2923 (2010)

29. V Genc, S Murphy, Y Yang, J Murphy, IEEE $802.16 \mathrm{~J}$ relay-based wireless access networks: an overview. IEEE Commun Mag. 15(5), 56-63 (2008) 
30. V Tarokh, H Jafarkhani, AR Calderbank, Space-time block coding for wireless communications: performance results. IEEE J Select Areas Commun. 17(3), 451-460 (1999)

31. Q Li, XL Lin, J Zhang, W Roh, Advancement of MIMO technology in WiMAX: from IEEE 802.16d/e/j to 802.16m. IEEE Commun Mag. 47(6), 100-107 (2009)

32. Y Li, Pilot-symbol-aided channel estimation for OFDM in wireless systems. IEEE Trans Veh Technol. 49(4), 1207-1215 (2000)

33. L Tong, BM Sadler, M Dong, Pilot-assisted wireless transmissions: General model, design criteria, and signal processing. IEEE Signal Process Mag. 21, 12-25 (2004)

34. CS Patel, GL Stuber, Channel estimation for amplify and forward relay based cooperation diversity systems. IEEE Trans Wireless Commun. 6(6), 2346-2356 (2007)

35. Y Han, A Pandharipande, SH Ting, Cooperative spectrum sharing via controlled amplify-and-forward relaying. in Proceedings of IEEE International Symposium on PIMRC, Cannes, France, Sept 2008

36. M Dottling, B Raaf, J Michel, Efficient channel quality feedback schemes for adaptive modulation and coding of packet data. in Proceedings of IEEE Vehicular Technology Conference, Los Angeles, Sept. 2004

doi:10.1186/1687-1499-2011-10

Cite this article as: Bohara et al: An orthogonal spectrum sharing scheme for wireless sensor networks. EURASIP Journal on Wireless

Communications and Networking 2011 2011:10.

\section{Submit your manuscript to a SpringerOpen ${ }^{\mathcal{O}}$ journal and benefit from:}

- Convenient online submission

- Rigorous peer review

- Immediate publication on acceptance

- Open access: articles freely available online

- High visibility within the field

- Retaining the copyright to your article

Submit your next manuscript at $\gg$ springeropen.com 